\title{
Inorganic Phosphate Modulates the Expression of the NaPi-2a Transporter in the trans-Golgi Network and the Interaction with PIST in the Proximal Tubule
}

\author{
Miguel A. Lanaspa, ${ }^{1,2}$ Yupanqui A. Caldas, ${ }^{1,2}$ Sophia Y. Breusegem, ${ }^{1}$ \\ Ana Andrés-Hernando, ${ }^{1,2}$ Christina Cicerchi, ${ }^{1}$ Moshe Levi, ${ }^{1}$ and Victor Sorribas ${ }^{2}$ \\ ${ }^{1}$ Department of Medicine, University of Colorado, and VA Eastern Colorado Health Care System, Denver, CO 80220, USA \\ ${ }^{2}$ Department of Toxicology, University of Zaragoza, 50013 Zaragoza, Spain
}

Correspondence should be addressed to Victor Sorribas; sorribas@unizar.es

Received 31 August 2012; Accepted 8 January 2013

Academic Editor: Habib Boukerche

Copyright (C) 2013 Miguel A. Lanaspa et al. This is an open access article distributed under the Creative Commons Attribution License, which permits unrestricted use, distribution, and reproduction in any medium, provided the original work is properly cited.

Inorganic phosphate $(\mathrm{Pi})$ homeostasis is maintained by the tight regulation of renal $\mathrm{Pi}$ excretion versus reabsorption rates that are in turn modulated by adjusting the number of Pi transporters (mainly NaPi-2a) in the proximal tubules. In response to some hormones and a high dietary Pi content, NaPi-2a is endocytosed and degraded in the lysosomes; however, we show here that some $\mathrm{NaPi}$-2a molecules are targeted to the trans-Golgi network (TGN) during the endocytosis. In the TGN, NaPi-2a interacts with PIST (PDZ-domain protein interacting specifically with TC10), a TGN-resident PDZ-domain-containing protein. The extension of the interaction is proportional to the expression of NaPi-2a in the TGN, and, consistent with that, it is increased with a high Pi diet. When overexpressed in opossum kidney (OK) cells, PIST retains NaPi-2a in the TGN and inhibits Na-dependent Pi transport. Overexpression of PIST also prevents the adaptation of OK cells to a low Pi culture medium. Our data supports the view that $\mathrm{NaPi}-2 \mathrm{a}$ is subjected to retrograde trafficking from the plasma membrane to the TGN using one of the machineries involved in endosomal transport and explains the reported expression of NaPi-2a in the TGN.

\section{Introduction}

The proximal tubule of the kidney is the main anatomical structure that maintains inorganic phosphate $(\mathrm{Pi})$ homeostasis, by virtue of its ability to fine-tune the Pi reabsorption rate. Renal Pi excretion and reabsorption depend on the abundance of specific $\mathrm{Na}$-dependent Pi transporters expressed in the brush-border membrane of the proximal tubular epithelial cells [1]. This abundance is controlled by several hormonal and nonhormonal agents, including phosphatonins, parathyroid hormone (PTH), and dietary Pi concentration [2]. Two type II Pi transporters (NaPi-2a and $\mathrm{NaPi}-2 \mathrm{c}$ ) and two type III transporters (PiT-1 and PiT-2) are expressed in the proximal tubule of the nephron. Of them, $\mathrm{NaPi}-2 \mathrm{a}$ is responsible for more than $90 \%$ of Pi reabsorption under standard conditions, as estimated using physiological approaches [3]. NaPi-2a has also been the most characterized $\mathrm{Pi}$ transporter, and the cellular mechanisms that regulate the apical expression of $\mathrm{NaPi}-2 \mathrm{a}$ in response to $\mathrm{PTH}$ or dietary $\mathrm{Pi}$ concentration have been extensively studied.

Acute changes in reabsorption rate are achieved within minutes by the fast insertion into, or retrieval of, Pi transporters from the membrane, mainly NaPi-2a. Potential posttranslational modifications, such as phosphorylation that could explain this regulatory mechanism, have not been described in the case of NaPi-2a. For example, upon binding of PTH to corresponding PTH receptors of proximal tubular cells, most NaPi-2a transporters are endocytosed through clathrin-coated pits and early endosomes and degraded in lysosomes $[4,5]$. This internalization is dependent on the rearrangement of microtubules into dense bundles of apicalto-basal orientation [6]. Microtubules are also involved in 
another acute regulation mechanism, that is, the adaptation to acute changes in dietary $\mathrm{Pi}$ concentration, but this mechanism has been less studied than the effect of PTH on the renal $\mathrm{Pi}$ transporters [7]. In rodents the expression of $\mathrm{NaPi}$ $2 \mathrm{a}$ in the brush border membrane of the proximal tubular cells increases within a few hours in response to low dietary Pi diet and decreases after eating high Pi-containing diets [8]. Changes in Pi transport activity and protein abundance of NaPi-2a during adaptation are not very extreme, but they are limited to $100 \%$ changes approximately, between minimal and maximal levels of both transport activity and protein abundance [9]. Acute upregulation in response to low Pi diet is independent of transcriptional and translational activities, suggesting the existence of an NaPi-2a storage mechanism that still has to be determined [7]. Similarly to the response to $\mathrm{PTH}$, a high Pi diet induces an acute downregulation of $\mathrm{Pi}$ reabsorption which is mediated by the fast internalization of $\mathrm{NaPi}-2 \mathrm{a}$ transporters from the brush border toward early and late endosomes and, again, lysosomal degradation [10]. In all these cases, the translocation of $\mathrm{NaPi}-2 \mathrm{a}$ transporters seems to be dependent on the interaction of NaPi-2a carboxyl-end with PDZ domain-containing proteins [11, 12].

The existence of intracellular storage of NaPi-2a has not deserved much attention. Immunofluorescence microscopy analyses have revealed clear colocalization of $\mathrm{NaPi}-2 \mathrm{a}$ with lysosomal and Golgi markers and additional subapical accumulations [7]. The relative expression in the different intracellular compartments varied with the Pi content of the diet, with maximal changes in expression corresponding to the lysosomal compartment after an acute (2-4 hours) adaptation from low-to-high Pi diet. More recently, however, we have described the changes in the expression in the transGolgi network (TGN) in parallel to the changes in dietary Pi [13].

In this work we have studied the in vivo expression of NaPi-2a in the TGN. Expression in TGN changes not only with dietary $\mathrm{Pi}$ adaptation, but also by overexpression of a TGN-located PDZ-domain-containing protein, the PDZ-domain protein interacting specifically with TC10, PIST $^{\text {CAL, GOPC, FIG }}$ [14]. Our findings suggest that NaPi-2a can be subjected to retrograde transport from the apical membrane toward the TGN, a novel mechanism that has been described for different membrane proteins, as an intermediate checkpoint step in the pathway to the degradation in the lysosomes [15].

\section{Materials and Methods}

2.1. Animal. Acute and chronic adaptations to different Pi diets have been detailed in numerous previous manuscripts (e.g., $[8,16])$. Briefly, rats were fed chronically (three days) fodder containing $0.1 \%$ (low), $0.6 \%$ (control), or $1.2 \%$ (high) Pi diets (Provimi Kliba SA, Penthalaz, Switzerland). Experiments involving animals were performed by the authors in Spain, according to European Animal Welfare regulations, and were explicitly approved by the ethical committee of the University of Zaragoza.
For acute experiments, male Wistar rats (6-8 week old; Janvier SAS, St. Berthevin, France) were supplied food from 08:00 to 10:00 AM each day, after which they had an access to tap water only. They were first conditioned on the high $\mathrm{Pi}$ or low Pi diets for 3 days, then switched to the acute dietary regime at 08:00 am (i.e., acute low-to-high $\mathrm{Pi}$, or acute highto-low $\mathrm{Pi}$ ), and subsequently sacrificed in triplicates four hours after switching to the acute diet.

2.2. Cell Culture and Transfections. Wild-type Opossum Kidney $(\mathrm{OK})$ cells were grown to confluence in DMEM/Ham'sF12 (Invitrogen, Carlsbad, CA, USA), 10\% FBS, as described in [13]. Transfections and cotransfections were achieved with Lipofectamine 2000 (Invitrogen) when the cells were 90\% confluent, following the manufacturer's instructions.

2.3. Transport Assays. Radiotracer uptake assays were performed using ${ }^{32} \mathrm{P}_{-} \mathrm{H}_{3} \mathrm{PO}_{4}$ as radiotracer, as reported previously $[13,17]$. Cells were grown on plastic plates, and uptake was measured in the presence and absence of $\mathrm{Na}^{+}$; in this case, $\mathrm{Na}^{+}$was substituted equimolarly by choline.

2.4. Immunoblotting and Coimmunoprecipitation Assays. Western blots were performed as described (e.g., [16]). Proteins were transferred into PVDF membranes and detected using either a polyclonal antibody against $\mathrm{NaPi}-2 \mathrm{a}$ from rat [16], or an anti-PIST (ProSci Inc., Poway, CA, USA) polyclonal antibody.

Coimmunoprecipitation was performed as reported in [18], using the same anti-PIST antibody as for immunodetection and a ProFound Mammalian Coimmunoprecipitation kit (Pierce, Rockford, IL, USA). Brush-border membranes (BBMs) from renal proximal tubular cells were prepared by the $\mathrm{Mg}^{2+}$ differential precipitation procedure [19]. For samples containing non-BBM proteins, the pellet obtained by $\mathrm{Mg}^{2+}$-precipitation after the first centrifugation was used.

2.5. Immunofluorescence Analysis. Rats were fixed in vivo by retrograde perfusion, as described in $[7,13]$. The transGolgi network was detected using a monoclonal anti- $\beta$ COP antibody (Sigma), $\beta$-actin using a goat anti- $\beta$-actin antibody (Santa Cruz Biotechnology, Santa Cruz, CA, USA), and tubulin using a monoclonal anti- $\alpha$-tubulin antibody (Sigma). For fluorescence microscopic imaging in OK cells, the open reading frame of rat $\mathrm{NaPi}-2 \mathrm{a}$ and rat PIST was cloned in frame, by high fidelity PCR cloning, into the fluorescent protein-encoding plasmids pEGFP-C1 and pECFPC1, respectively, (both from Clontech, Mountain View, CA, USA). Immunofluorescent preparations of either tissue sections or OK cells were analyzed with a laser-scanning confocal microscope LSM510 (Carl Zeiss, Thornwood, NY, USA).

2.6. Statistical Analysis. Data were compared with Student's $t$-test for two experimental groups. Results were expressed as Mean \pm SD. All statistical analysis was performed with GraphPad Prism 5.0 statistical software. 

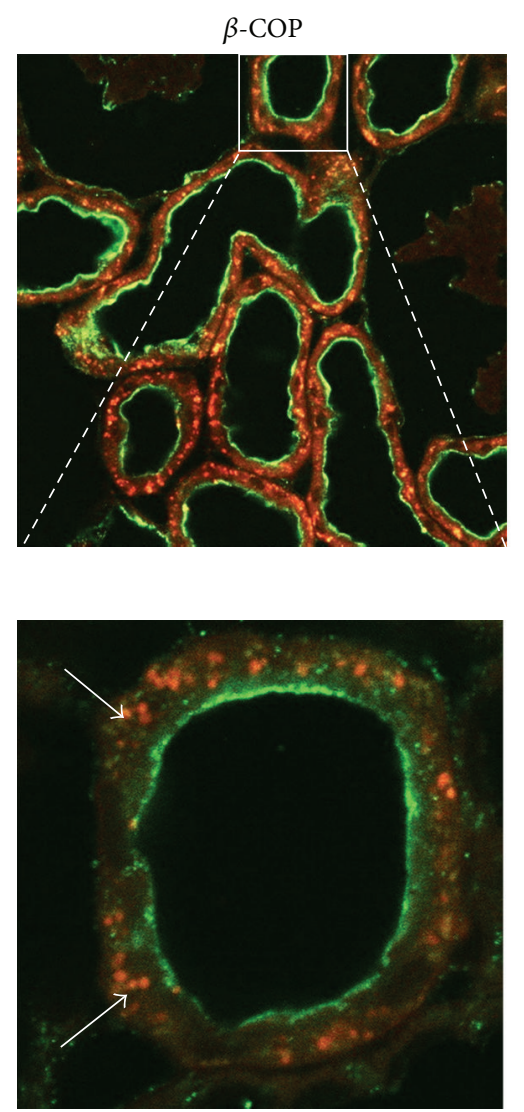

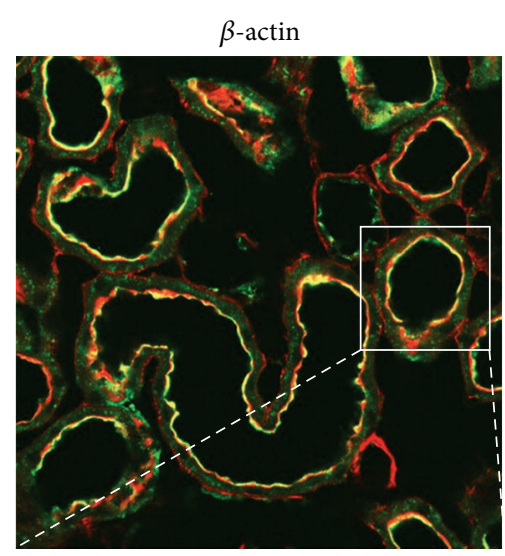

(a)

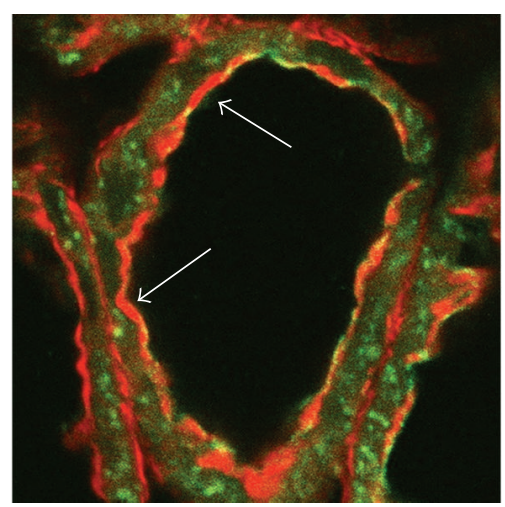

(b)
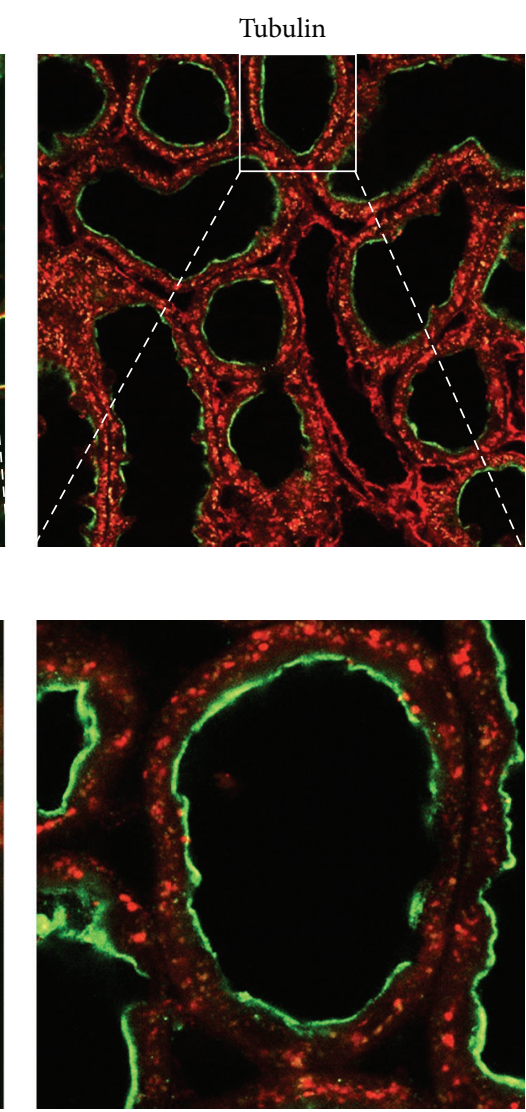

FIgURE 1: Confocal immunodetection in rat kidney cortex of NaPi-2a (green, Alexa488), $\beta$-COP (red, Alexa568), $\beta$-actin (red, phalloidinrhodamine), and tubulin (red, Alexa 568). NaPi-2a colocalizes with $\beta$-COP intracellularly, and with $\beta$-actin in the BBM (see arrows, orange merge). NaPi-2a does not colocalize with tubulin (only green and red are visible, under steady-state conditions). (a) Low magnification of kidney cortex; (b) high magnification to show single proximal tubules.

\section{Results}

3.1. Expression of $\mathrm{NaPi}$-2a in trans-Golgi Network. In addition to the main expression of $\mathrm{NaPi}-2 \mathrm{a}$ in the brush-border membrane of proximal tubular cells, this Pi transporter also exhibits a perinuclear staining, which is compatible with a specific expression in the trans-Golgi network [7, 13]. Consequently, we first checked whether the intracellular expression of $\mathrm{NaPi}-2 \mathrm{a}$ could be colocalized with $\beta$-COP, a classical marker of the trans-Golgi. In animals fed a control Pi diet $(0.6 \% \mathrm{Pi})$, immunofluorescence of rat kidney sections showed that, as expected, $\mathrm{NaPi}-2 \mathrm{a}$ was mostly expressed in the brushborder membrane of the proximal tubular epithelial cells, but intracellular staining could also be observed (Figure 1). The intracellular expression colocalized completely with $\beta$-COP. Thus, under this dietary control condition the intracellular expression of NaPi-2a could now be defined as the transGolgi network.

3.2. Colocalization of NaPi-2a with PIST in the trans-Golgi Network. NaPi-2a expression and subcellular localization are dependent on the interaction of the NaPi-2a carboxyl end with PDZ-domain-containing proteins, such as NHERF1 and PDZK1. PIST is a PDZ protein that localizes mostly to the trans-Golgi network, so we sought to determine whether interaction with PIST could explain the expression of NaPi-2a in the TGN. To facilitate the expression analysis the study was performed under two dietary conditions known to modify the expression of $\mathrm{NaPi}-2 \mathrm{a}$ in the apical membrane: 24 hours adaptation to low (0.1\%) and high (1.2\%) Pi-containing diets. As expected, under Pi deprivation conditions ( $0.1 \% \mathrm{Pi}$ diet) practically all signal of the immunofluorescent-labeled NaPi2a was visualized in the brush-border membrane, and no colocalization with the PIST protein could be observed either in the brush border or in intracellular localizations (Figure 2). However, when the animals were chronically fed a high $\mathrm{Pi}$ content diet (1.2\% Pi), NaPi-2a was very weakly expressed in the apical membrane, but the intracellular staining of this Pi transporter was increased, showing a clear, but partial colocalization with PIST (Figure 2; see arrows).

3.3. Interaction of NaPi-2a and PIST. Based on the colocalization of NaPi-2a and PIST observed in rat kidney by IF, and on the fact that several interactions between membrane proteins and the PDZ domain of PIST have been described during the last decade [20-23], we next checked whether NaPi-2a was 

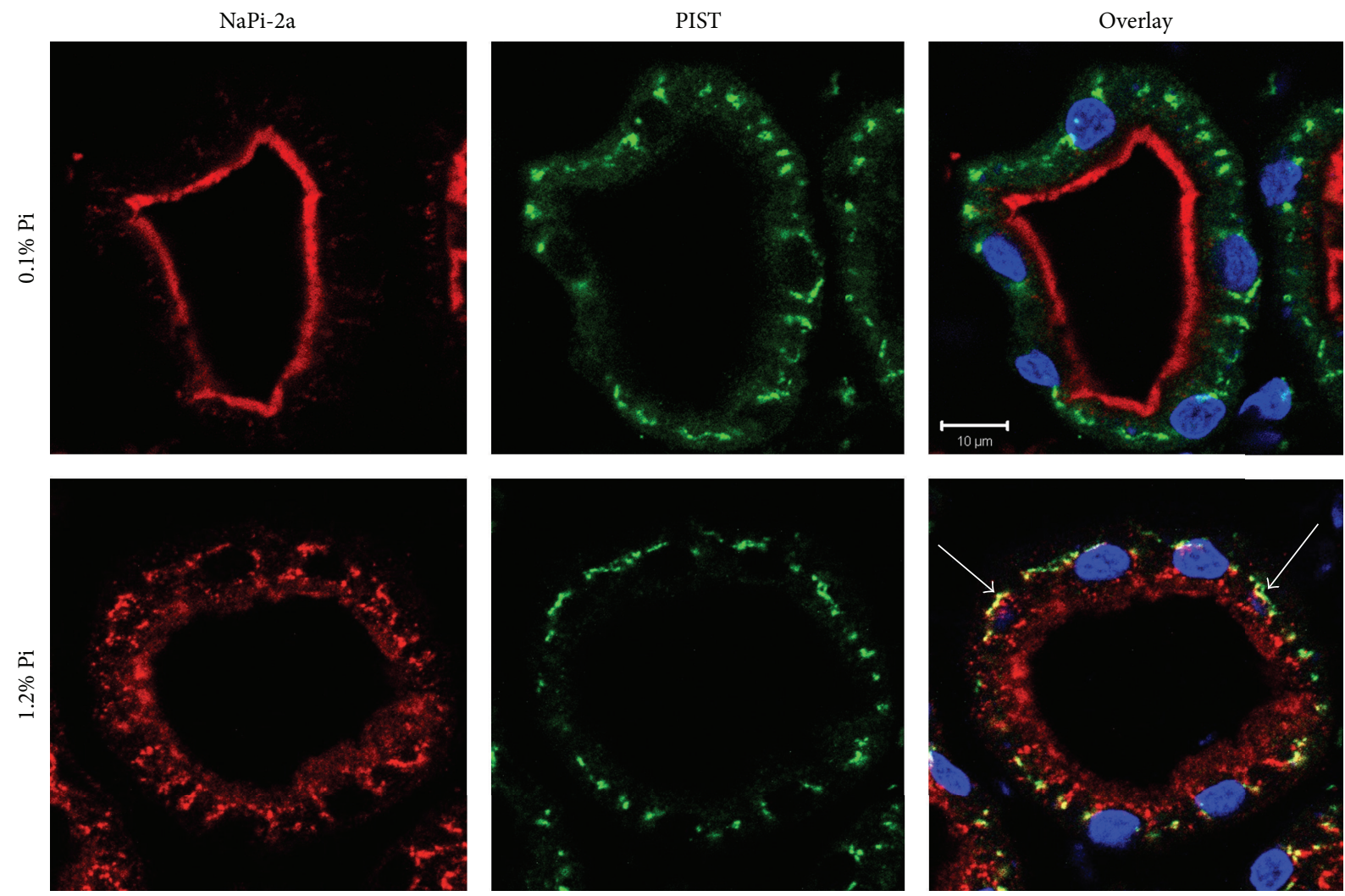

Figure 2: Expression of NaPi-2a (red) and PIST (green) in rat proximal tubules fed chronically (24h) a low (0.1\%) or high (1.2\%) Pi diet. Colocalization only takes place intracellularly in high Pi diet fed animals (arrows). Nuclei stained with DAPI (blue).

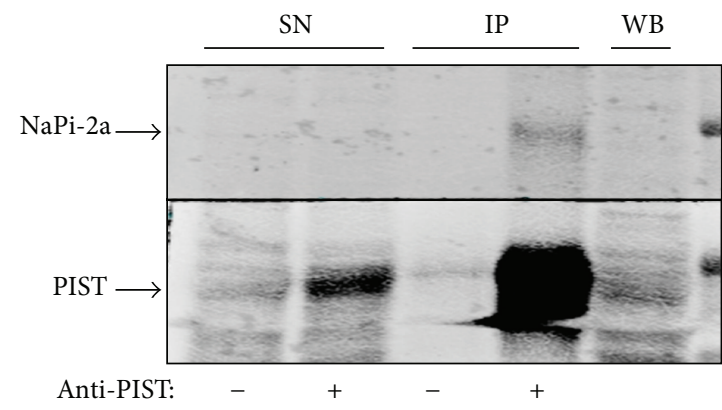

(a)

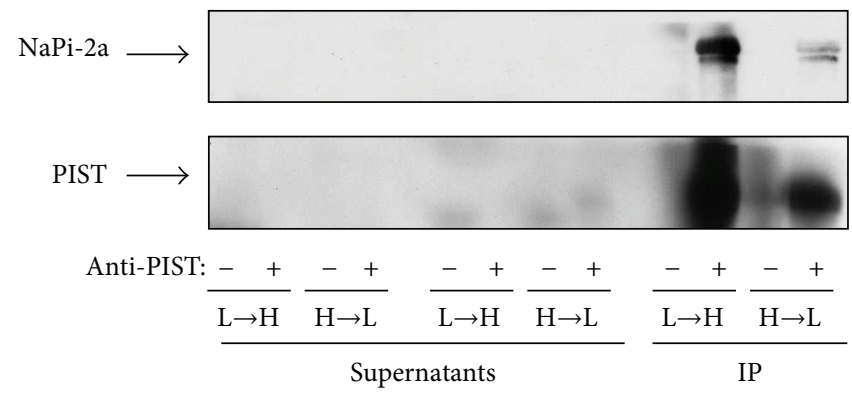

(b)

FIgURE 3: Coimmunoprecipitation assays. (a) Coimmunoprecipitation of PIST and NaPi2a using rat kidney homogenates from rats fed a control diet, and with an anti-PIST antibody. (b) Coimmunoprecipitation in rats acutely adapted for 4 hours from low to high (L-H) and from high to low (H-L) Pi diet, using non-BBM homogenates. SN: supernatant (nonprecipitated samples); IP: immunoprecipitated; WB: Western blotting.

also able to interact with this PDZ protein. Coimmunoprecipitation assays were performed using a polyclonal anti-PIST antibody and rat kidney samples. Initially, coimmunoprecipitation was performed in the total homogenate of kidney cortex from control diet $(0.6 \% \mathrm{Pi})$ fed rats (Figure $3(\mathrm{a})$ ). Under these conditions, a weak band specific to NaPi-2a was observed, therefore suggesting an interaction between the transporter and PIST. Interaction was then determined under acute adaptations ( 4 hours only) to the two Pi diets, in order to study not only the steady state conditions established during the chronic adaptations, but also the movements from and to the cell membrane. Figure 3(b) shows the results of the two conditions that were studied: from chronic low $\mathrm{Pi}$ to acute high $\mathrm{Pi}$ diets (low-to-high, $0.1 \%$ to $1.2 \%$ ) and from chronic high $\mathrm{Pi}$ to acute low $\mathrm{Pi}$ diets (high-to-low, $1.2 \%$ to $0.1 \%$ ), following the standard procedure explained in Section 2 (e.g., [9]). After the four hours of acute adaptation the interaction was assayed, revealing that the immunoprecipitated $\mathrm{NaPi}$ 2a was more abundant in the low-to-high Pi diet adaptation than in the high-to-low Pi diet; that is, the interaction was 


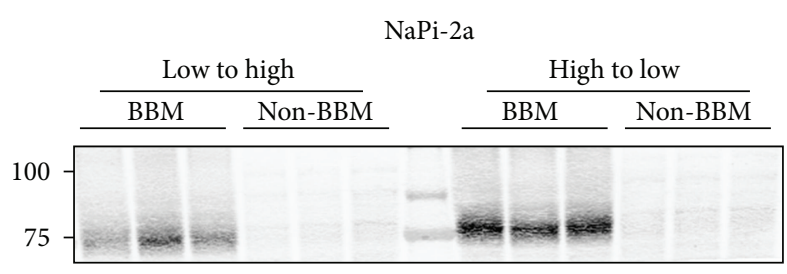

(a)

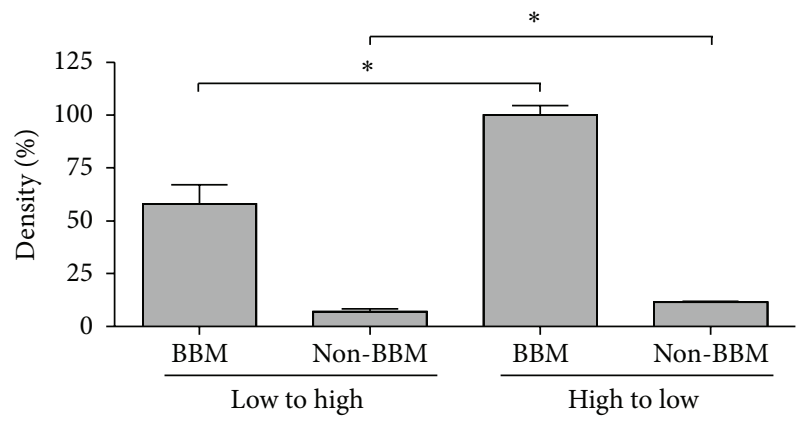

(b)

Figure 4: Expression of NaPi-2a using immunoblots of BBM and non-BBM. Bottom chart, corresponding densitometries of the immunosignals with statistical differences.

favored when $\mathrm{NaPi}$-2a protein is internalized from the brushborder membrane as an adaptation to an increased Pi intake (Figure 3(b)). It is important to clarify that while several proteins are described to interact with the PDZ domain of PIST through their C-termini (see above), we have not demonstrated that this is the case for $\mathrm{NaPi}-2 \mathrm{a}$, and, therefore, other molecular interactions can occur.

A Western blot was also performed to determine the abundance of NaPi-2a in the brush-border membrane (BBM) and the non-BBM fractions (Figure 4). Western blot confirmed that $\mathrm{NaPi}-2 \mathrm{a}$ was mainly expressed in the brush border membranes of animals adapted from high-to-low Pi diet, and with minor expression in non-BBM membranes. The low expression is mainly based on the absence of transGolgi membrane purifications, because the combination of all nonbrush-border membranes was used as samples.

3.4. Effect of PIST Overexpression on $\mathrm{NaPi}-2 a$ Expression in OK Cells. The effect of PIST overexpression was evaluated in Opossum Kidney (OK) cells, a classical in vitro model of proximal tubular cells. Transfection of a CFP-NaPi-2a fusion protein plasmid in OK cells reveals that the protein is mainly expressed in the brush-border membrane of the cells, with some minor expression intracellularly, which is compatible with a trans-Golgi network localization. When CFP-NaPi-2a is cotransfected with a GFP-PIST plasmid, both fluorescent fusion proteins colocalize intracellularly, again in a place close to the nucleus and compatible with TGN, which in turn, is the normal expression location of PIST (Figure 5(a)).

$\mathrm{OK}$ cells express an endogenous $\mathrm{NaPi}-2 \mathrm{a}$ transporter, $\mathrm{NaPi}-4$ [24], which is usually expressed in the brush-border membrane, and intracellular expression is minimal under steady-state conditions (Figure 5(b)). When OK cells were single-transfected with GFP-PIST, resulting in the overexpression of this PDZ protein, NaPi-4 disappeared from the membrane and colocalized with GFP-PIST (Figure 5(c)). Consequently, transfection of PIST also inhibited $\mathrm{Na}$ dependent Pi transport by $40 \%$ approximately compared to mock transfected cells, a percentage that is similar to the transfection efficiency of the plasmid (Figure 5(d)).

OK cells are an established model for $\mathrm{Pi}$ studies that have the ability to adapt to the concentration of $\mathrm{Pi}$ in the culture medium similarly to the proximal tubular cells. In the presence of low $\mathrm{Pi}$ concentration, the expression of $\mathrm{NaPi}$ 4 in the apical membrane increases rapidly $[9,25]$ as in the mammalian kidney (Figure 2). However, when GFP-PISTtransfected $\mathrm{OK}$ cells were adapted acutely (for two hours) by incubating them to either low $(0.1 \mathrm{mM})$ or to high $(2 \mathrm{mM})$ $\mathrm{Pi}$ culture medium starting from the control condition, $1 \mathrm{mM}$ Pi, the classical adaptation was blunted by the PIST overexpression. In other words, in PIST-transfected OK cells, $\mathrm{NaPi}-4$ was maintained in the TGN, independently of the concentration of Pi in the culture medium (Figure 6), without the adaptation response of the cells.

\section{Discussion}

Pi reabsorption rate in the renal proximal tubule is the main target mechanism for all hormonal and nonhormonal signals that control $\mathrm{Pi}$ homeostasis. Reabsorption rate is determined by the abundance of $\mathrm{Pi}$ transporters (mainly $\mathrm{NaPi}$ 2a) in the brush-border membrane. Consequently, because $\mathrm{Pi}$ transporters seem to be always active and functional (i.e., they are not phosphorylated), changes in Pi transport rate are only the consequence of insertion of transporters into, or internalization from the brush-border membrane. Internalization takes place through endocytosis via clathrincoated pits and early endosomes, as a response to PTH, the phosphatonins (e.g., FGF23), or adaptation to high Pi diet $[4,5,7]$. The final outcome of the endocytosed transporters is degradation into lysosomes, but this process has not been studied in great detail. The present work is the starting point in a project aimed to study the precise outcome of this $\mathrm{Pi}$ transporter after endocytosis-mediated internalization and the role that the PDZ-domain protein PIST might play in this process.

NaPi-2a interacts through its carboxyl end with several PDZ-domain-containing proteins, such as NHERF-1 and PDZK1 (NHERF3), but the role of these interactions in the subcellular expression and localization in the cell is also not completely understood. NHERF-1 facilitates the residence of $\mathrm{NaPi}-2 \mathrm{a}$ in the brush-border membrane [26], and it is necessary for the adaptation to a low Pi diet [12]. The role of PDZK1 is less evident, but the corresponding RNA is increased during chronic dietary restriction of $\mathrm{Pi}$ [24]. The specific role that differentiates PDZK1 from NHERF-1 interaction with $\mathrm{NaPi}-2 \mathrm{a}$ is not clear, beyond a mayor scaffold role beneath the apical membrane [18].

Here we are describing a new interaction between NaPi$2 \mathrm{a}$ and a trans-Golgi resident PDZ protein, PIST [14]. We 

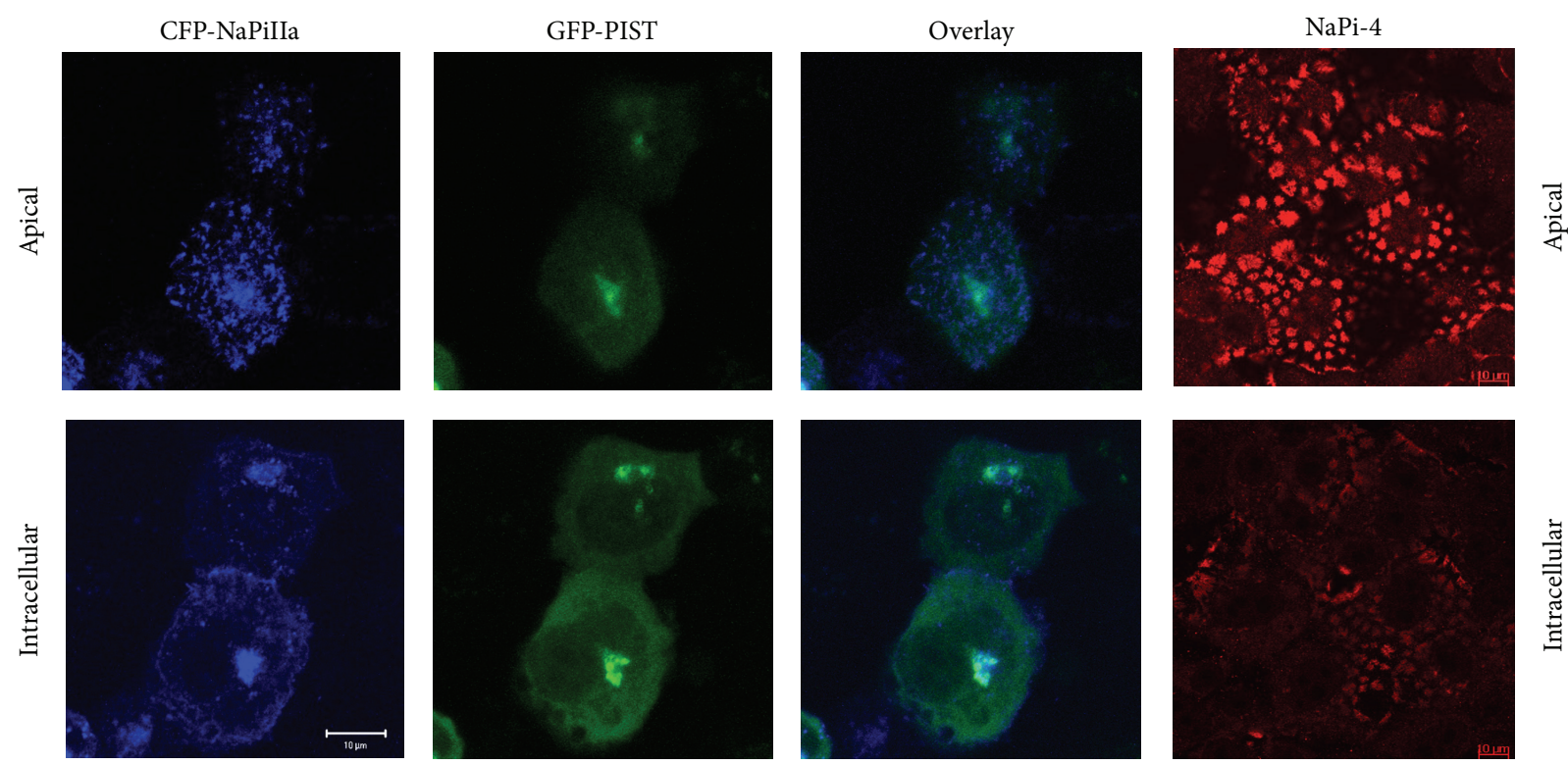

(a)

(b)
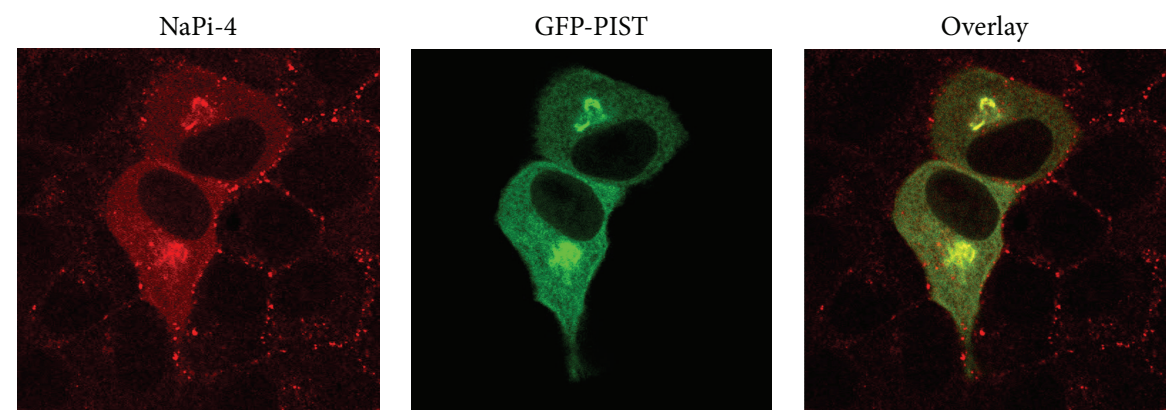

(c)

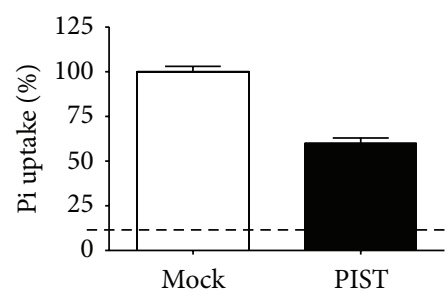

(d)

FIGURE 5: (a) Coexpression of CFP-NaPi2a and GFP-PIST in OK cells. The two proteins colocalize intracellularly at the site of PIST expression. (b) Expression of the endogenous NaPi-2a in OK cells ( NaPi-4) is only clearly seen in BBM under control conditions. (c) Overexpression of PIST restricts the expression of NaPi4 to the TGN. (d) Pi transport in OK cells transfected with GFP-PIST is reduced proportionally to the efficiency of PIST transfection. Dashed line: level of Na-independent uptake.

have described this interaction using an in vivo approach, to avoid the misinterpretations that overexpression (in vitro) frequently causes. However, in vivo studies have many limitations in understanding the cellular handling of the transporters, and, therefore, parallel in vitro cell culture studies are also necessary. With these studies we have shown that the interaction with PIST explains the expression of NaPi-2a in the TGN $[7,13]$, but also that both the extension of the interaction and the expression of NaPi-2a in TGN, are modified by the $\mathrm{Pi}$ content of the diet (Figures 2 and 3 ). We are not showing direct evidence of the interaction between NaPi-2a and PIST through a PDZ domain interaction. Nevertheless, this is most likely the case, as NaPi-2a almost exclusively interacts with other proteins through its PDZ-binding domain, and the PDZ interacting proteins are the same as other membrane proteins with similar carboxy ends (see below, the CFTR case).

The expression of NaPi-2a in the TGN and the interaction with PIST that we are describing complement the knowledge about the intracellular trafficking of the transporter in the proximal tubular cell. The role of the TGN in Pi homeostasis is of major interest because the TGN is one of the main proteintrafficking checkpoints of the cell at the crossroads between exocytic and endocytic pathways. The TGN, located at the exit face of the Golgi apparatus, sorts cargo proteins (and lipids) into carrier systems towards the plasma membrane, endosomes, and so forth, but it also receives proteins from various endosomal locations, by a process known as retrograde transport $[15,27]$. Retrograde transport can occur from the early endosome, the recycling endosome, or from late endosome, and the choice of one or more routes depends on the specific cargo. The different cargos require specific machineries, including the newly described retromer [28]. The TGN decides whether the endocytosed protein is going to either be degraded through the ubiquitin proteasome or through the lysosome pathways, or recycled to the plasma membrane using the recycling endosome. In the case of $\mathrm{NaPi}$ $2 \mathrm{a}$, it is not degraded by the proteasome but by the lysosome 

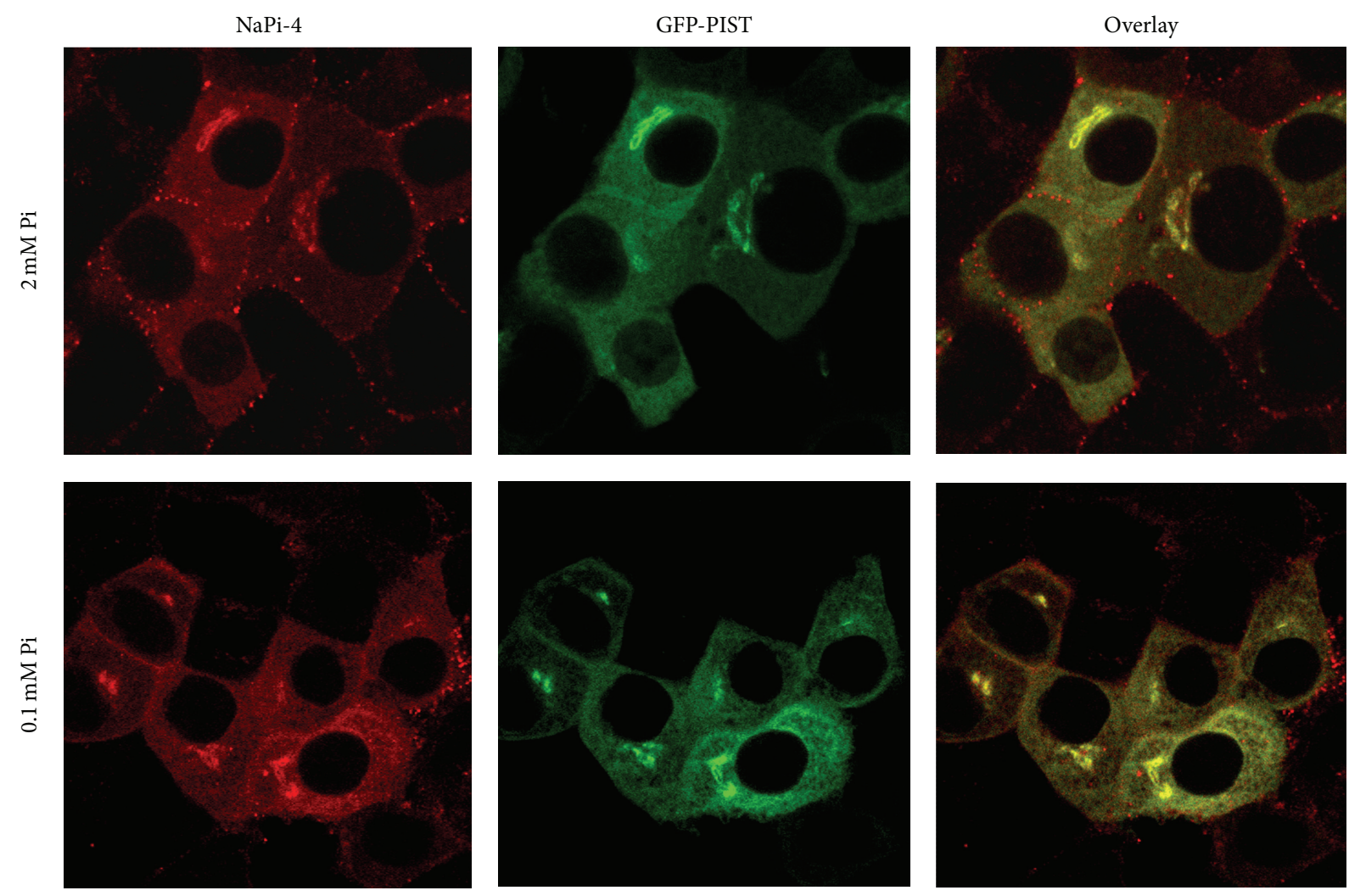

FIGURE 6: Overexpression of GFP-PIST in OK cells maintains NaPi4 in the trans-Golgi, independently of the concentration of Pi in the cell culture medium $(0.1 \mathrm{mM}$ versus $2 \mathrm{mM} \mathrm{Pi})$.

$[7,10]$. However, the existence of a low recycling rate back to the plasma membrane has not been discarded, and therefore the traffic through the TGN should be analyzed in detail.

It seems that for the transition through the TGN the interaction with PIST is a mandatory step, because there is a correlation between the abundance of $\mathrm{NaPi}-2 \mathrm{a}$ expression in the TGN (Figure 2; [14]) and the intensity of the NaPi2a/PIST interaction (Figure 3). In addition, overexpression of PIST in OK cells induces the internalization of $\mathrm{NaPi}-2 \mathrm{a}$ to the TGN and therefore inhibits Pi transport (Figure 5), but, most important, overexpression also impedes the cells to adapt acutely to a low Pi culture medium (Figure 6). This suggests that the insertion of $\mathrm{NaPi}-2 \mathrm{a}$ transporters from intracellular storage (i.e., adaptation to low $\mathrm{Pi}$ concentration) also involves a sorting through the TGN or, alternatively, that overexpression of PIST is impairing the correct functioning of the cargo machinery involved in the movement of $\mathrm{NaPi}-2 \mathrm{a}$ transporters to the plasma membrane. The interaction of PIST seems to be specific with $\mathrm{NaPi}-2 \mathrm{a}$ among Pi transporters, because $\mathrm{NaPi}-2 \mathrm{c}$ does not interact with PIST, and $\mathrm{NaPi}-2 \mathrm{c}$ is not expressed in the TGN [29]. With respect to type III Pi transporters (PiT1 and PiT-2), they do not contain PDZ-binding domains at their carboxy ends, and the intracellular expression is located in the endoplasmic reticulum [30]. Also, the acute adaptation of $\mathrm{NaPi}-2 \mathrm{a}$ is faster and very different than that of $\mathrm{NaPi}-2 \mathrm{c}$ and
PiT-2 [16, 31], a phenomenon that can also be explained by the existence of $\mathrm{NaPi}-2 \mathrm{a}$ recycling.

The step through the TGN and the interaction with PIST has also been described for different membrane proteins, with different outcome [19-22]. For example, the cystic fibrosis transmembrane conductance regulator (CFTR) interacts with the same PDZ-domain proteins as NaPi-2a (NHERF1, NHERF-2, PDZK1, and PIST; [19]). When endocytosed, CFTR can be recycled back to the plasma membrane or trafficked to the lysosome, and this depends, at least in part, on the interaction of the carboxyl end of CFTR with the PDZ proteins. In contrast to NHERF-1, PIST reduces cell surface expression of CFTR, retains it in the cell, and promotes degradation in the lysosome [32]. PIST seems to be a component of the trafficking machinery that connects the plasma membrane and the TGN. For this purpose, PIST also needs to interact with a variety of proteins such as TC10 (a Rho family small GTPase) or syntaxin 6 (a member of the Q-SNARE family; [33]). We do not know whether $\mathrm{NaPi}-2 \mathrm{a}$ behaves similarly to CFTR, but it is tempting to suggest that not all NaPi-2a is degraded into the lysosomes as a response to PTH or high Pi concentration, as it would imply a high energetic cost to the cell. Whether NaPi-2a is degraded or recycled, we do not know clearly yet, but in any of both outcomes the TGN will be, most likely, the 
subcellular structure that will dictate the trafficking route of the internalized transporters.

\section{Conflict of Interests}

The authors reveal that there are no conflict of interests in the present paper.

\section{Authors' Contribution}

M. A. Lanaspa and Y. A. Caldas contributed equally to this work.

\section{Acknowledgments}

This work was supported by two grants from the regional government of Aragon, code PI020/09 and from the Spanish Ministry of Science and Innovation (Grant no. BFU2009$12763 / \mathrm{BFI})$, both to V. Sorribas, and by a National Institute of Health, 2R01 DK066029-06, to M. Levi.

\section{References}

[1] I. Forster, N. Hernando, V. Sorribas, and A. Werner, "Phosphate transporters in renal, gastrointestinal, and other tissues," Advances in Chronic Kidney Disease, vol. 18, no. 2, pp. 63-76, 2011.

[2] J. Marks, E. S. Debnam, and R. J. Unwin, "Phosphate homeostasis and the renal-gastrointestinal axis," American Journal of Physiology, vol. 299, no. 2, pp. F285-F296, 2010.

[3] R. Villa-Bellosta and V. Sorribas, "Compensatory regulation of the sodium/phosphate cotransporters NaPi-IIc (SCL34A3) and Pit-2 (SLC20A2) during Pi deprivation and acidosis," Pflugers Archiv: European Journal of Physiology, vol. 459, no. 3, pp. 499508, 2010.

[4] D. Bacic, M. LeHir, J. Biber, B. Kaissling, H. Murer, and C. A. Wagner, "The renal Na+/phosphate cotransporter NaPi-IIa is internalized via the receptor-mediated endocytic route in response to parathyroid hormone," Kidney International, vol. 69, no. 3, pp. 495-503, 2006.

[5] M. F. Pfister, I. Ruf, G. Stange et al., "Parathyroid hormone leads to the lysosomal degradation of the renal type II Na/Pi cotransporter," Proceedings of the National Academy of Sciences of the United States of America, vol. 95, no. 4, pp. 1909-1914, 1998.

[6] M. Lötscher, Y. Scarpetta, M. Levi et al., "Rapid downregulation of rat renal $\mathrm{Na} / \mathrm{P}(\mathrm{i})$ cotransporter in response to parathyroid hormone involves microtubule rearrangement," The Journal of Clinical Investigation, vol. 104, no. 4, pp. 483-494, 1999.

[7] M. Lötscher, B. Kaissling, J. Biber, H. Murer, and M. Levi, "Role of microtubules in the rapid regulation of renal phosphate transport in response to acute alterations in dietary phosphate content," The Journal of Clinical Investigation, vol. 99, no. 6, pp. 1302-1312, 1997.

[8] M. Levi, M. Lotscher, V. Sorribas et al., "Cellular mechanisms of acute and chronic adaptation of rat renal $\mathrm{P}(\mathrm{i})$ transporter to alterations in dietary P(i)," American Journal of Physiology, vol. 267, no. 5, pp. F900-F908, 1994.

[9] R. Villa-Bellosta and V. Sorribas, "Different effects of arsenate and phosphonoformate on $\mathrm{P}_{i}$ transport adaptation in opossum kidney cells," American Journal of Physiology, vol. 297, no. 3, pp. C516-C525, 2009.

[10] I. Keusch, M. Traebert, M. Lötscher, B. Kaissling, H. Murer, and J. Biber, "Parathyroid hormone and dietary phosphate provoke a lysosomal routing of the proximal tubular $\mathrm{Na} / \mathrm{Pi}$-cotransporter type II," Kidney International, vol. 54, no. 4, pp. 1224-1232, 1998.

[11] P. Capuano, D. Bacic, G. Stange et al., "Expression and regulation of the renal $\mathrm{Na}$ /phosphate cotransporter $\mathrm{NaPi}$-IIa in a mouse model deficient for the PDZ protein PDZK1," Pflugers Archiv: European Journal of Physiology, vol. 449, no. 4, pp. 392402, 2005.

[12] E. J. Weinman, A. Boddeti, R. Cunningham et al., "NHERF1 is required for renal adaptation to a low-phosphate diet," American Journal of Physiology, vol. 285, no. 6, pp. F1225-F1232, 2003.

[13] M. A. Lanaspa, H. Giral, S. Y. Breusegem et al., "Interaction of MAP17 with NHERF3/4 induces translocation of the renal $\mathrm{Na} / \mathrm{Pi}$ IIa transporter to the trans-Golgi," American Journal of Physiology, vol. 292, no. 1, pp. F230-F242, 2007.

[14] C. L. Neudauer, G. Joberty, and I. G. Macara, "Pist: a novel pdz/coiled-coil domain binding partner for the rho-family gtpase tc10," Biochemical and Biophysical Research Communications, vol. 280, no. 2, pp. 541-547, 2001.

[15] Z. Z. Lieu and P. A. Gleeson, "Endosome-to-Golgi transport pathways in physiological processes," Histology and Histopathology, vol. 26, no. 3, pp. 395-408, 2011.

[16] R. Villa-Bellosta, S. Ravera, V. Sorribas et al., "The $\mathrm{Na}^{+}-$ $\mathrm{P}_{i}$ cotransporter PiT-2 (SLC20A2) is expressed in the apical membrane of rat renal proximal tubules and regulated by dietary $\mathrm{P}_{i}$," American Journal of Physiology, vol. 296, no. 4, pp. F691-F699, 2009.

[17] V. Sorribas, D. Markovich, G. Hayes et al., "Cloning of a $\mathrm{Na} / \mathrm{P}(\mathrm{i})$ cotransporter from opossum kidney cells," The Journal of Biological Chemistry, vol. 269, no. 9, pp. 6615-6621, 1994.

[18] S. M. Gisler, S. Pribanic, D. Bacic et al., "PDZK1: I. A major scaffolder in brush borders of proximal tubular cells," Kidney International, vol. 64, no. 5, pp. 1733-1745, 2003.

[19] J. Biber, B. Stieger, G. Stange, and H. Murer, "Isolation of renal proximal tubular brush-border membranes," Nature Protocols, vol. 2, no. 6, pp. 1356-1359, 2007.

[20] J. Cheng, B. D. Moyer, M. Milewski et al., "A golgi-associated $\mathrm{PDZ}$ domain protein modulates cystic fibrosis transmembrane regulator plasma membrane expression," The Journal of Biological Chemistry, vol. 277, no. 5, pp. 3520-3529, 2002.

[21] M. Gentzsch, L. Cui, A. Mengos, X. B. Chang, J. H. Chen, and J. R. Riordan, "The PDZ-binding chloride channel ClC-3B localizes to the Golgi and associates with cystic fibrosis transmembrane conductance regulator-interacting PDZ proteins," The Journal of Biological Chemistry, vol. 278, no. 8, pp. 64406449, 2003.

[22] W. Wente, T. Stroh, A. Beaudet, D. Richter, and H. J. Kreienkamp, "Interactions with PDZ domain proteins PIST/GOPC and PDZK1 regulate intracellular sorting of the somatostatin receptor subtype 5," The Journal of Biological Chemistry, vol. 280, no. 37, pp. 32419-32425, 2005.

[23] Z. Xu, K. Oshima, and S. Heller, "PIST regulates the intracellular trafficking and plasma membrane expression of Cadherin 23," BMC Cell Biology, vol. 11, article 80, 2010.

[24] M. Custer, B. Spindler, F. Verrey, H. Murer, and J. Biber, "Identification of a new gene product (diphor-1) regulated by dietary phosphate," American Journal of Physiology, vol. 273, no. 5, pp. F801-F806, 1997. 
[25] D. Markovich, T. Verri, V. Sorribas, J. Forgo, J. Biber, and H. Murer, "Regulation of opossum kidney (OK) cell $\mathrm{Na} / \mathrm{P}_{i}$ cotransport by $\mathrm{P}_{i}$ deprivation involves mRNA stability," Pflugers Archiv: European Journal of Physiology, vol. 430, no. 4, pp. 459463, 1995.

[26] S. Shenolikar, J. W. Voltz, C. M. Minkoff, J. B. Wade, and E. J. Weinman, "Targeted disruption of the mouse NHERF1 gene promotes internalization of proximal tubule sodiumphosphate cotransporter type IIa and renal phosphate wasting," Proceedings of the National Academy of Sciences of the United States of America, vol. 99, no. 17, pp. 11470-11475, 2002.

[27] L. Johannes and V. Popoff, "Tracing the retrograde route in protein trafficking," Cell, vol. 135, no. 7, pp. 1175-1187, 2008.

[28] M. E. Harbour, S. Y. A. Breusegem, R. Antrobus, C. Freeman, E. Reid, and M. N. J. Seaman, "The cargo-selective retromer complex is a recruiting hub for protein complexes that regulate endosomal tubule dynamics," Journal of Cell Science, vol. 123, no. 21, pp. 3703-3717, 2010.

[29] R. Villa-Bellosta, M. Barac-Nieto, S. Y. Breusegem, N. P. Barry, M. Levi, and V. Sorribas, "Interactions of the growth-related, type IIc renal sodium/phosphate cotransporter with PDZ proteins," Kidney International, vol. 73, no. 4, pp. 456-464, 2008.

[30] R. Villa-Bellosta, M. Levi, and V. Sorribas, "Vascular smooth muscle cell calcification and SLC20 inorganic phosphate transporters: effects of PDGF, TNF- $\alpha$, and Pi," Pflugers Archiv: European Journal of Physiology, vol. 458, no. 6, pp. 1151-1161, 2009.

[31] H. Segawa, S. Yamanaka, M. Ito et al., "Internalization of renal type IIc Na- $\mathrm{P}_{i}$ cotransporter in response to a high-phosphate diet," American Journal of Physiology, vol. 288, no. 3, pp. F587F596, 2005.

[32] J. Cheng, H. Wang, and W. B. Guggino, "Modulation of mature cystic fibrosis transmembrane regulator protein by the PDZ domain protein CAL," The Journal of Biological Chemistry, vol. 279, no. 3, pp. 1892-1898, 2004.

[33] J. Cheng, V. Cebotaru, L. Cebotaru, and W. B. Guggino, "Syntaxin 6 and CAL mediate the degradation of the cystic fibrosis transmembrane conductance regulator," Molecular Biology of the Cell, vol. 21, no. 7, pp. 1178-1187, 2010. 

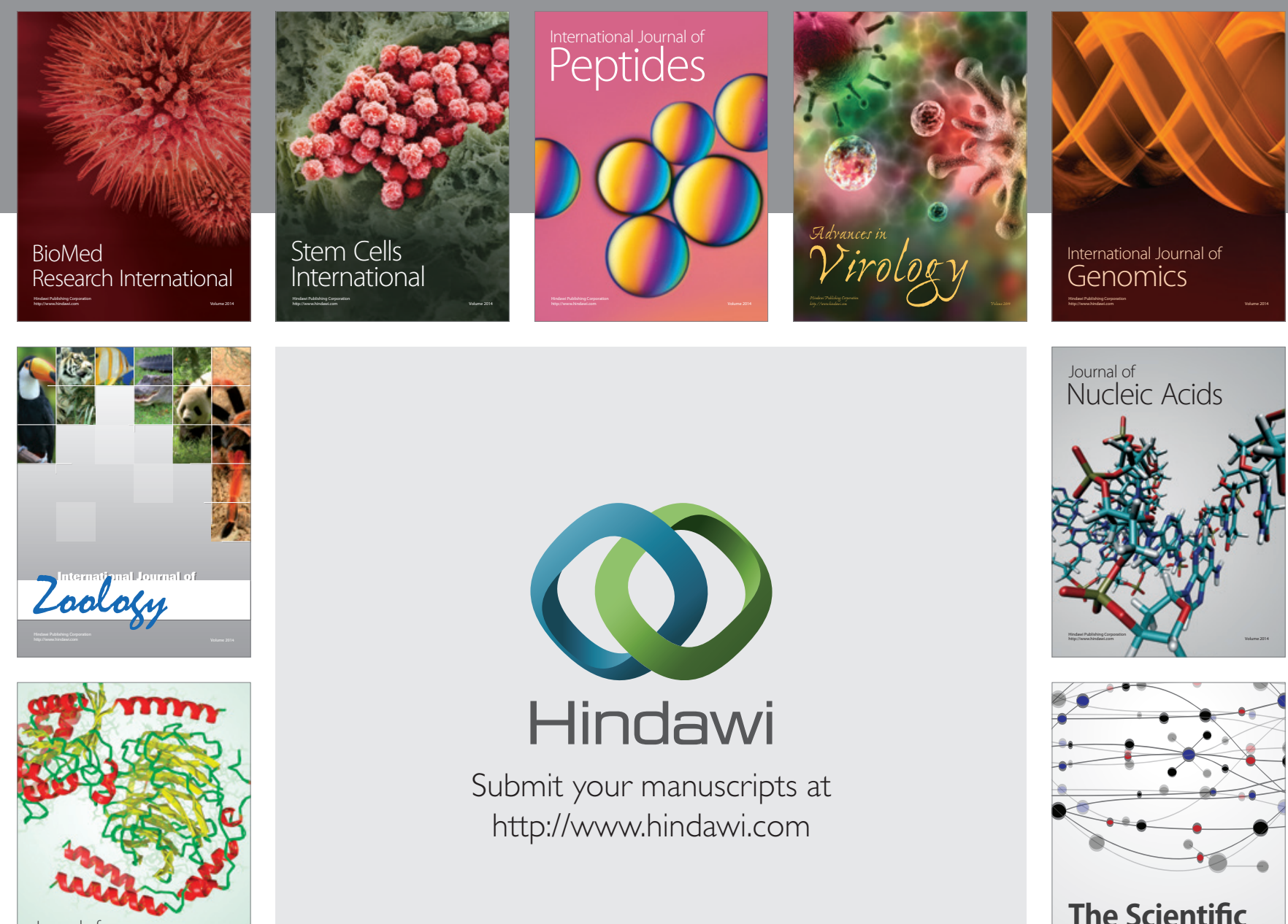

Submit your manuscripts at

http://www.hindawi.com

Journal of
Signal Transduction
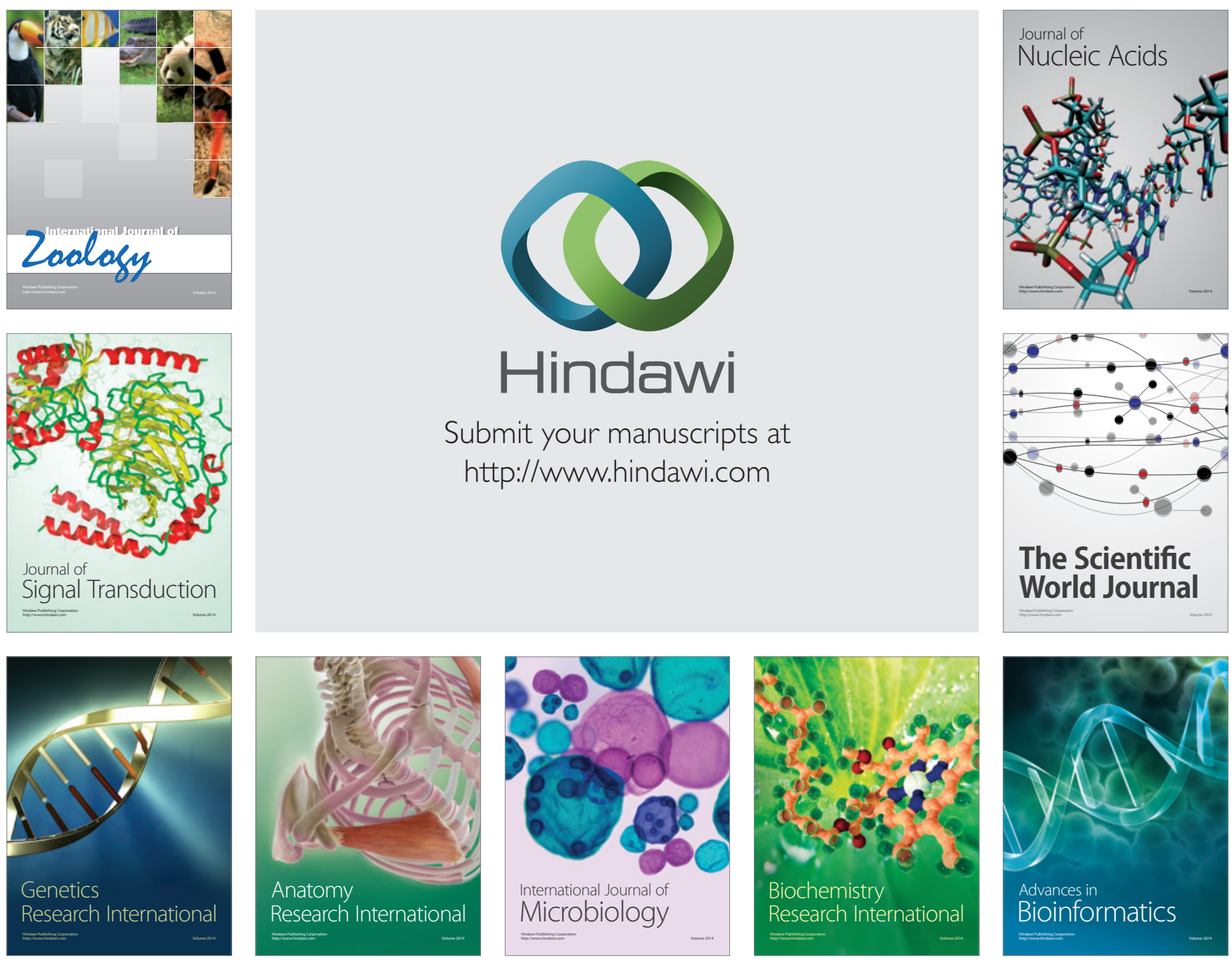

The Scientific World Journal
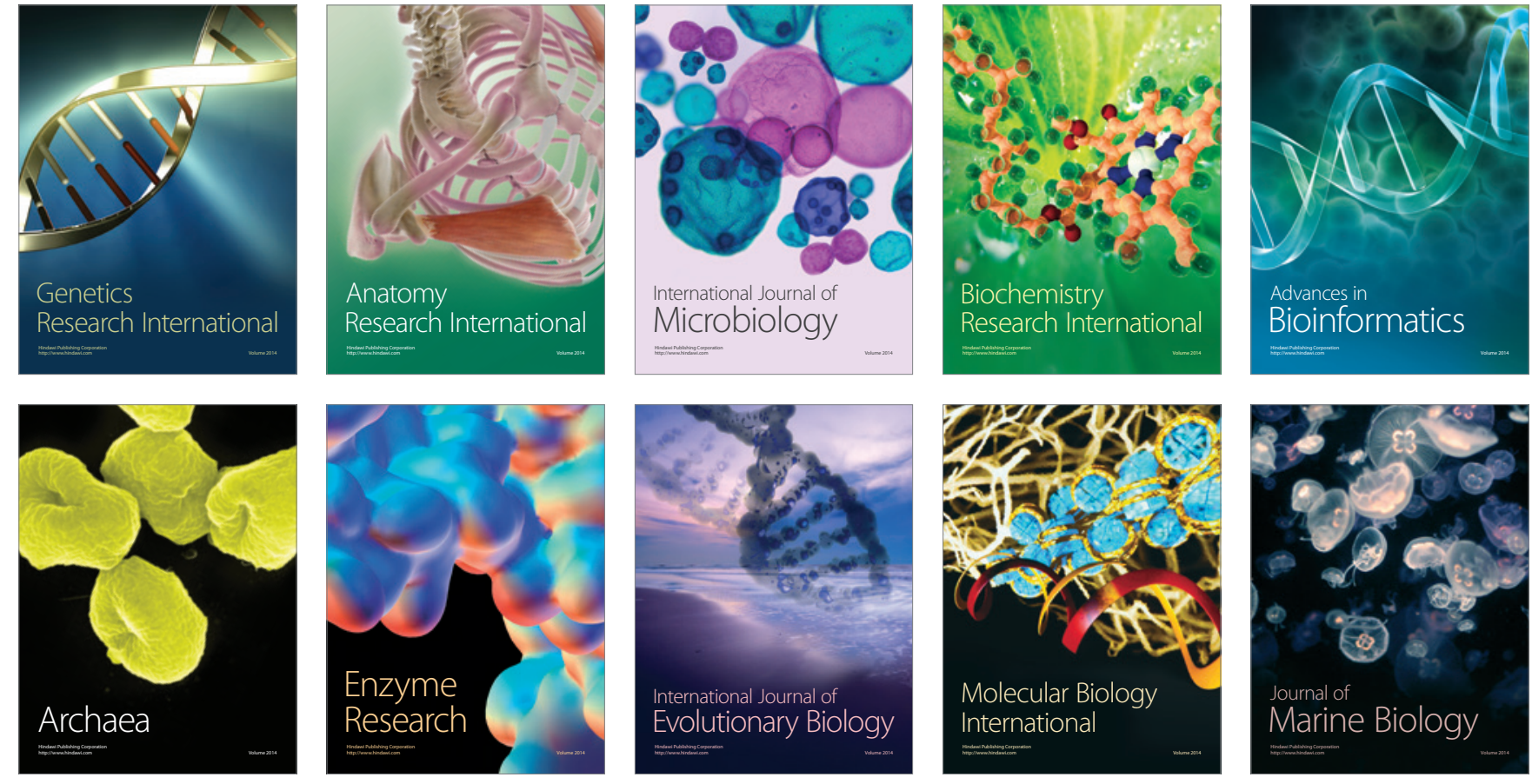